\title{
Only half of the mothers practiced early initiation of breastfeeding in Northwest Ethiopia, 2015
}

\author{
Amare Tariku ${ }^{*} \mathbb{C}$, Gashaw Andargie Biks², Molla Mesele Wassie ${ }^{1}$, Abebaw Gebeyehu Worku ${ }^{3}$ \\ and Melaku Kindie Yenit ${ }^{4}$
}

\begin{abstract}
Background: Early initiation of breastfeeding has been well-recognized in reducing neonatal mortality; however, it remains sub-optimal in Ethiopia. This study therefore assessed the prevalence of early initiation of breastfeeding and associated factors among mothers with children aged 6-24 months in Dabat Health and Demographic Surveillance System (HDSS) site, northwest Ethiopia, where literature on the issue is markedly scarce.

Methods: This community-based cross-sectional survey was carried out from May to June, 2015, at Dabat HDSS site, Dabat District. Eight hundred twenty-two mother-child pairs were included in the study. A multivariable logistic regression model was employed to identify factors associated with early initiation of breastfeeding.

Results: This study demonstrated that the prevalence of early initiation of breastfeeding was $53.3 \%$. Institutional delivery ( $\mathrm{AOR}=4.9 ; 95 \% \mathrm{Cl} 3.2,7.4)$, higher Infant and Young Child Feeding (IYCF) knowledge (AOR $=2.3 ; 95 \% \mathrm{Cl}$ 1.6, 3.3), higher wealth status ( $A O R=4.1,95 \% \mathrm{Cl} 2.8,6.0$ ) and low fathers' education ( $\mathrm{AOR}=0.3,95 \% \mathrm{Cl} 0.2,0.6)$ were significantly associated with early initiation of breastfeeding in the multivariate analysis.

Conclusion: In summary, the coverage of early initiation of breastfeeding in Dabat HDSS site was low, considerably below the national target. Therefore, efforts should be intensified to step-up early initiation of breastfeeding by focusing on the identified determinants.
\end{abstract}

Keywords: Early initiation of breastfeeding, Children aged 6-24 months, Health and Demographic Surveillance System, Ethiopia

\section{Background}

Breastfeeding is a well-recognized child survival strategy [1] as it has been proved in reducing the risk of different childhood diseases, including diarrhea and acute respiratory tract infections [1-4]. Particularly, a significant reduction in neonatal mortality is documented with initiation of breastfeeding within $1 \mathrm{~h}$ after birth, commonly named Early Initiation of Breastfeeding (EIBF) $[1,3,5-8]$. On the other hand, EIBF improves lactation and uterine contraction following delivery [7]. In spite of

\footnotetext{
*Correspondence: amaretariku15@yahoo.com

1 Department of Human Nutrition, Institute of Public Health, College of Medicine and Health Sciences, University of Gondar, Gondar, Ethiopia Full list of author information is available at the end of the article
}

these priceless benefits, EIBF is sub-optimally practiced in many countries of the world [4]. EIBF practice ranges from 22 to $63 \%$ in Africa, [9-12], while it is $42.2-83.3 \%$ in Asia $[13,14]$. Surprisingly, only one in ten $(11.4 \%)$ mothers practiced EIBF in Saudi Arabia [15]. The coverage of EIBF is 35.2 and $47.1 \%$ in Turkey and Brazil, respectively $[16,17]$.

Likewise, the prevalence of EIBF is found low in Ethiopia; nationally $52 \%$ of the mothers practice EIBF [7]. Some of the district level studies also illustrate low prevalence of EIBF as it was shown in Jimma Arijo (62.6\%) [18], Goba (52.4\%) [19], Debre Markos (51.8\%) [20] and Arbaminch (42.8\%) [21]. However, majority of mothers initiated breastfeeding early in Nekemte District (88.5\%) [22] and East Wollega Zone (83.3\%) [23]. 
Based on the findings of former researches high EIBF practice was noted among mothers who had better infant and young child feeding (IYFC) knowledge [18, 20, 2327], antenatal care (ANC) follow up, got breastfeeding counseling $[9,16,24]$, and gave birth at health facilities $[9,12,14,28,29]$. Improved mothers' education [10, 18, $19,21,30]$ and socioeconomic status [9, 24, 30, 31] also increases the liklihood of EIBF practice.

Nationally, different strategies have been implemented in Ethiopia to enhance infant and child feeding practice, including EIBF [32-34], nevertheless most of the infants still feed sub-optimally [7]. In addition, researches are limited. Therefore, to fill the knowledge gap, this study investigated EIBF and associated factors among mothers who had children aged 6-24 months at Dabat Health and Demographic Surveillance System (HDSS) site.

\section{Methods}

This cross-sectional survey was carried out from May to June 2015 in Dabat HDSS site situated in Dabat District, Ethiopia. This surveillance site was instituted in 1996 and currently it expands its scope of investigation by including 13 kebeles (smallest administrative units). A total of 67,385 people are living in the site. The details of information about the surveillance site is available elsewhere $[35,36]$.

This study is part of the big survey entitled 'Child Nutritional Status and Feeding Practice'. All children (6-59 months) with their mothers living in the HDSS site were participated in above survey. Representative eight kebeles were chosen through lottery method, then all mother-child pairs living in these kebeles were included. However, mothers with less than 6 months of residency were excluded from the survey. Epi-info version 3.7 was used to estimate a sample size of 804 by considering the expected proportion of mothers practicing EIBF as $52 \%$ [7], 95\% confidence level, $5 \%$ margin of error, $5 \%$ non-response rate and a design effect of 2 . However, we included all 822 mother-child pairs fulfilling the criteria for analysis to enhance the power of the study.

A structured and pretested questionnaire was used to collect data. Seventeen research assistants (14 data collectors and 3 supervisors) were recruited for the survey. English version questionnaire was translated to Amharic, the native language of the study area, and retranslated to English. Research assistants were trained and the tool was piloted before the actual data collection. EIBF, the outcome variable, is defined as putting the newborn to the breast within 1 hour of birth [1]. However, most of the mothers reported the time at which the infant received breast milk rather than telling the time at which they first put the newborn to the breast because they misunderstood the question [37]. Therefore, the study participants were asked how long after birth they first put [name] to the breast even if their breast milk did not arrive early enough.

Concerning the measurement of explanatory variables, household wealth status and mother's infant and young child feeding knowledge were estimated using principal component analysis (PCA). For both variables, factor scores were summed and ranked into terciles (poor, medium and high). Further information about the items and approaches used for dependent variables ascertainment is available from the previous publication [35].

EPI-Info version 3.5.3 was used for data entry, while Statistical Package for Social Sciences (SPSS) version 20 analysis used for analysis. Hence clustering effect was not detected among kebeles of the HDSS site, individual level analysis was carried out. A binary logistic regression model was fitted to identify factors associated with EIBF. Initially bivariable analysis was done to show the independent effect of each explanatory variable on the outcome variable, EIBF. A variable selection criteria of $\mathrm{p}$ values of $<0.2$ in the bivariable analysis was used for the final model, consequently all variables fulfilling the criteria (mothers and fathers' education and employment status, mothers IYCF knowledge, place of delivery, health care access and household wealth status) were fitted to the multivariable logistic regression model. Significance of association was declared at a $\mathrm{p}$ value of $<0.05$. The strength of association was shown using crude odds ratio (COR) and adjusted odds ratio (AOR) with a 95\% confidence intervals. Multi-collinearity was checked, considering the variance inflation factor (VIF), none of the independent variables were co-linear. The Hosmer and Lemeshow goodness of fit-test was estimated at 0.74 , suggesting the model fitted the data well.

\section{Results}

The mean ( \pm Standard Deviation, SD) age of children was $17.3( \pm 7.3)$ months. Nearly three-quarters $(67.4 \%$ and $69.5 \%$, respectively) of the fathers and mothers were illiterate (Table 1). About $37.0 \%$ of participants had no history of ANC follow up and $75.9 \%$ gave birth at home (Table 2). This study demonstrated that the prevalence of EIBF was 53.3\% [95\% CI: 49.8, 56.7]. Moreover, the result of multivariate logistic regression analysis illustrated that place of delivery, mother's IYCF knowledge, household wealth status, and father's educational status were significantly and independently associated with EIBF.

Accordingly, higher odds of EIBF were noted among mothers who gave birth at a health facility $[\mathrm{AOR}=4.9$; $95 \%$ CI 3.2, 7.4] compared to those who did it at home. Mothers with medium $[\mathrm{AOR}=2.1 ; 95 \% \mathrm{CI} 1.4,3.1]$ and high [AOR: 2.3; 95\% CI 1.6, 3.3] IYCF knowledge had increased odds of EIBF compared to mothers with poor 
Table 1 Socio-demographic and economic characteristics of the study participants in Dabat HDSS site, Dabat District, northwest Ethiopia, 2015

\begin{tabular}{|c|c|c|c|c|c|}
\hline \multirow{2}{*}{\multicolumn{3}{|c|}{ trict, northwest Ethiopia, 2015}} & \multirow{2}{*}{\multicolumn{2}{|c|}{$\begin{array}{ll}\text { Variables } & \text { Frequency }\end{array}$}} & \multirow[b]{2}{*}{ Percent } \\
\hline & & & & & \\
\hline Variables & Frequency & Percent & \multicolumn{3}{|l|}{ Antenatal care visits } \\
\hline \multicolumn{3}{|l|}{ Child age (months) } & No visit & 304 & 37.0 \\
\hline $6-11$ & 239 & 29.1 & 1 & 54 & 6.6 \\
\hline $12-24$ & 583 & 70.9 & $2-3$ & 286 & 34.8 \\
\hline \multicolumn{3}{|l|}{ Child sex } & $\geq 4$ & 178 & 21.6 \\
\hline Male & 410 & 49.9 & Place of delivery & & \\
\hline Female & 412 & 50.1 & Home & 624 & 75.9 \\
\hline \multicolumn{3}{|l|}{ Religion } & Health facilities & 198 & 24.1 \\
\hline Orthodox & 773 & 94.0 & Delivery attendant & & \\
\hline Other $^{a}$ & 49 & 6.0 & Health professionals & 203 & 24.7 \\
\hline \multicolumn{3}{|l|}{ Mother's age } & Traditional birth attendant & 153 & 18.6 \\
\hline $15-19$ & 46 & 5.6 & Relatives and volunteers & 466 & 56.7 \\
\hline $20-34$ & 461 & 56.1 & Postnatal follow-up & & \\
\hline$\geq 35$ & 315 & 38.3 & Yes & 212 & 25.8 \\
\hline \multicolumn{3}{|l|}{ Mother's education } & No & 610 & 74.2 \\
\hline Illiterate & 571 & 69.5 & Health care access & & \\
\hline Literate & 251 & 30.5 & Good access & 607 & 73.8 \\
\hline \multicolumn{3}{|l|}{ Mother's employment } & Poor access & 215 & 26.2 \\
\hline Housewife & 482 & 58.6 & Health extension visit in the past 6 months & & \\
\hline Farmer & 211 & 25.7 & No visit & 391 & 47.5 \\
\hline Other ${ }^{b}$ & 129 & 15.7 & $1-2$ visit & 327 & 39.8 \\
\hline \multicolumn{3}{|l|}{ Mother's marital status } & $\geq 3$ & 104 & 12.7 \\
\hline Currently married & 739 & 89.9 & Mother's IYCF ${ }^{a}$ knowledge & & \\
\hline Currently unmarried $^{c}$ & 83 & 10.1 & Poor & 272 & 33.1 \\
\hline \multicolumn{3}{|l|}{ Father's education } & Medium & 285 & 34.7 \\
\hline Illiterate & 554 & 67.4 & High & 268 & 32.2 \\
\hline Primary school & 143 & 17.4 & Prelacteal feeding given to the child & & \\
\hline Secondary school and above & 125 & 15.2 & Yes & 220 & 26.8 \\
\hline \multicolumn{3}{|l|}{ Father's employment } & No & 602 & 73.2 \\
\hline Unemployed & 46 & 5.6 & a Infant and Young Child Feeding & & \\
\hline
\end{tabular}

Farmer

Other employment ${ }^{\mathrm{d}}$

52.4

42.0

Household size

$\begin{array}{lll}\leq 4 & 304 & 37.0 \\ >4 & 518 & 63.0\end{array}$

Number of children under 5 years

$$
1
$$$$
2-3
$$

Wealth status

Poor

Medium

High

${ }^{a}$ Muslim, protestant and catholic

b Students, unemployed, servant, own business

c Single, divorced and widowed

d Merchant, contract and permanent work
Table 2 Maternal health care utilization in Dabat HDSS site, Dabat District, northwest Ethiopia, 2015

IYCF knowledge. Likewise, the odds of EIBF were higher among mothers in medium $[\mathrm{AOR}=2.4,95 \% \mathrm{CI} 1.6,3.6$ ] and high wealth status categories [AOR $=4.1,95 \%$ CI 2.8, 6.0] compared to those who were in poor wealth status category. However, the odds of EIBF were lower among children whose fathers were either illiterate $[A O R=0.3$, $95 \%$ CI $0.2,0.6]$ or attended primary school $[\mathrm{AOR}=0.5$, 95\% CI 0.3, 0.9] than children whose fathers attended secondary school and above (Table 3).

\section{Discussion}

In the Health Sector Development Programme IV, Ethiopia set a target to raise EIBF practice to $92 \%$ by the end of 2015 [32]. However, this study demonstrated that 
Table 3 Factors associated with early initiation of breastfeeding among mothers who had children aged 6-24 months in Dabat HDSS site, Dabat District, northwest Ethiopia, 2015

\begin{tabular}{|c|c|c|c|c|}
\hline \multirow[t]{2}{*}{ Variables } & \multicolumn{2}{|c|}{ Early initiation of breastfeeding } & \multirow[t]{2}{*}{ Crude odds ratio $\left(95 \% \mathrm{Cl}^{* *}\right)$} & \multirow[t]{2}{*}{ Adjusted odds ratio $(95 \% \mathrm{Cl})$} \\
\hline & Yes (\#) & No (\#) & & \\
\hline \multicolumn{5}{|l|}{ Mother's education } \\
\hline Illiterate & 270 & 301 & 1 & 1 \\
\hline Literate & 114 & 137 & $1.1(0.8,1.5)$ & $1.1(0.7,1.3)$ \\
\hline \multicolumn{5}{|l|}{ Mother's employment } \\
\hline Farmer & 84 & 127 & $1.4(0.9,2.0)$ & $0.8(0.4,1.5)$ \\
\hline Housewife & 272 & 210 & 1 & 1 \\
\hline Others & 82 & 47 & $0.8(0.4,1.5)$ & $1.4(0.9,2.0)$ \\
\hline \multicolumn{5}{|l|}{ Father's employment } \\
\hline Unemployed & 31 & 15 & $2.1(1.1,3.9)$ & $1.6(0.7,3.3)$ \\
\hline Farmer & 234 & 197 & $1.2(0.9,1.6)$ & $1.3(0.9,1.8)$ \\
\hline Others & 173 & 172 & 1 & 1 \\
\hline \multicolumn{5}{|l|}{ Father's education } \\
\hline Illiterate & 286 & 268 & $0.2(0.1,0.4)$ & $0.3(0.2,0.6)^{*}$ \\
\hline Primary school & 57 & 86 & $0.3(0.2,0.5)$ & $0.5(0.3,0.9)^{*}$ \\
\hline Secondary school and above & 95 & 30 & 1 & 1 \\
\hline \multicolumn{5}{|l|}{ Wealth status } \\
\hline Poor & 103 & 204 & 1 & 1 \\
\hline Medium & 172 & 97 & $3.5(2.5,4.6)^{*}$ & $2.4(1.6,3.6)^{*}$ \\
\hline High & 163 & 83 & $3.9(2.7,5.6)^{*}$ & $4.1(2.8,6.0)^{*}$ \\
\hline \multicolumn{5}{|l|}{ Mother's IYFC knowledge } \\
\hline Poor & 114 & 158 & 1 & 1 \\
\hline Medium & 155 & 110 & $2.0(1.4,2.8)^{*}$ & $2.1(1.4,3.1)^{*}$ \\
\hline High & 169 & 116 & $2.0(1.4,2.8)^{*}$ & $2.3(1.6,3.3)^{*}$ \\
\hline \multicolumn{5}{|l|}{ Place of delivery } \\
\hline Home & 283 & 341 & 1 & 1 \\
\hline Health facility & 155 & 43 & $4.4(3.0,6.3)^{*}$ & $4.9(3.2,7.4)^{*}$ \\
\hline \multicolumn{5}{|l|}{ Health care access } \\
\hline Good access & 313 & 294 & $0.8(0.6,1.1)$ & $0.9(0.6,1.4)$ \\
\hline Poor access & 125 & 90 & 1 & 1 \\
\hline
\end{tabular}

* Significant at a $p$ value of $<0.05$

** Confidence interval

only $53.5 \%$ of mothers initiated breastfeeding within one hour of birth. The finding was consistent with the 2011 Ethiopian Demographic Health Survey (DHS) report (52\%) [7] and other local studies done in Arbaminch and Goba districts which reported 57.2\% [21] and 52.4\% [19], respectively.

However, our finding was the lowest compared to findings from other parts of Ethiopia, for example, Nekemtie District (88.5\%) [22], East Wollega Zone, (83.5\%) [23], and Jimma Arjo District (63\%) [18]. The low prevalence of EIBF in the current study could be related to the lower utilization of institutional delivery compared to the studies done in Nekemtie District and East Wollega Zone. Other Previous reports claimed that institutional delivery was associated with a higher likelihood of EIBF [9,
$14,28]$. On the other hand, different studies documented that prelacteal feeding was higher among mothers who gave birth at home $[35,38]$ and this is associated with lower odds of EIBF [38, 39].

Findings similar to ours, for example, 46.1\% [11, 12], $56 \%$ [9], and $47.1 \%$ [16] were reported from Tanzania, Uganda, and Brazil, respectively. On the other hand, the prevalence of EIBF in our work was higher than the reports from Saudi Arabia (11.4\%) [15], India (22\%) [40], Bangladesh (46.3\%) [13], Nepal (42.2\%) [24], and Turkey (35.2\%) [17]. Maternal health care utilization and access to child feeding information were higher in the latter study areas $[15,40]$. Therefore, a lower prevalence of EIBF in the above overseas studies could be related to mothers' perception of the inadequate and delayed 
production of breast milk which forced them to give prelacteal feeds. In fact, mother's unfavorable perception of breastfeeding was related to delayed initiation and subsequent sub-optimal breastfeeding practices [41, 42].

The result of the adjusted analysis indicated that place of delivery, mother's IYCF knowledge, household wealth status, and fathers' education were significantly and independently associated with EIBF, while household wealth status was positively associated with it. The finding was supported by reports from some developing countries [ 9 , $24,30,31]$. This could be partially explained by the positive effect of socioeconomic status on mothers' health care utilization, institutional delivery, for instance. The Ethiopian DHS report (2011) also showed that mothers' institutional delivery preference was enhanced with the improvement of household socio-economic status [7].

Similarly, this study showed that the odds of EIBF were high among mothers who gave birth at health facilities. This was in agreement with the reports from other African and Asian countries [9, 12, 14, 28, 29]. Obviously, institutional delivery creates a chance to deliver skilled guidance/support about appropriate neonatal feeding information and serves as the only way to deliver immediate obstetric cares, including supporting mothers to initiate breastfeeding imminently after birth. Breastfeeding support and counseling by health care professionals is one of the crucial interventions to step-up early initiation into breastfeeding [43-46]. However, 43 (21.7\%) of the mothers who gave birth at health facilities delayed the initiation into breastfeeding. This could be related to the poor commitment of health care professionals to support mothers in practicing early initiation into breastfeeding. This however requires further investigations.

A lower paternal educational status (illiterate and primary school) was associated with decreased odds of EIBF compared to those who had higher educational status, secondary school and above. The finding was supported by studies done elsewhere $[19,21,24,28]$. In fact, paternal support and favorable attitude were affirmed in boosting mothers' confidence to practice optimal breastfeeding [42, 47, 48]. Also, education is a crucial tool in enhancing parent's awareness of appropriate neonatal feeding practices [7]. In line with earlier findings [20, 23, $24,26,27,49]$, this study illustrated that the odds of EIBF among mothers with higher IYCF knowledge were higher compared to those who had low IYCF knowledge. Stepping-up mothers IYCF knowledge is one of the key interventions to mitigate the high burden of inappropriate infant and child feeding practices [34]. Despite its importance, most of the national nutrition strategies which are designed to improve IYCF practice, did not adequately describe behavioral change and communication components [33, 34].
Our study showed the extent and factors associated with EIBF practice in the rural population of northwest Ethiopia where health care and other social services, including education, are not adequacy accessible. However, some limitation should be understood. Though efforts were made (as mentioned in the method section) to help the mother to remember the actual time of initiation of breastfeeding, the study is not free from recall bias. Hence, this study utilized a data collected to answer another research question; some of the other variables, such as birth weight were not included. Given that, selection bias is the other possible limitation of the study.

\section{Conclusion}

In summary, despite the fact that there are implementations of programs and strategies in Dabat HDSS site, the coverage of EIBF was considerably below the national target. The result also suggested that EIBF was associated with health care utilization and socio-economic factors. This implies that further improvements of the utilization of institutional delivery and paternal involvement are critical to enhance EIBF coverage. Also, increasing mothers' IYCF knowledge through strengthening breastfeeding education and counseling programs are essential. Finally, a longitudinal study approach is highly recommended for further investigations.

\section{Abbreviations \\ HDSS: Health and Demographic Surveillance System; AOR: adjusted odds ratio; COR: crude odds ratio; $\mathrm{Cl}$ : confidence interval; IYCF: infant and young child feeding; EIBF: early initiation of breastfeeding; WHO: World Health Organization; ANC: antenatal care; SPSS: Statistical Package for Social Sciences; SD: standard deviation.}

\section{Authors' contributions}

AT GAB MMW AGW Conceived and designed the experiments. GAB AT Performed the experiments. AT MKY analyzed the data. AT GAB MKY MMW AGW wrote the paper. All authors read and approved the final manuscript.

\section{Author details}

${ }^{1}$ Department of Human Nutrition, Institute of Public Health, College of Medicine and Health Sciences, University of Gondar, Gondar, Ethiopia. ${ }^{2}$ Department of Health Service Management and Health Economics, Institute of Public Health, College of Medicine and Health Sciences, University of Gondar, Gondar, Ethiopia. ${ }^{3}$ Department of Reproductive and Child Health, Institute of Public Health, College of Medicine and Health Sciences, University of Gondar, Gondar, Ethiopia. ${ }^{4}$ Department of Epidemiology and Biostatistics, Institute of Public Health, College of Medicine and Health Sciences, University of Gondar, Gondar, Ethiopia.

\section{Acknowledgements}

Authors would like to thank all mothers for their willingness to participate in the study. They are also grateful to the University of Gondar and Dabat Research Center for the financial and material support, respectively. Finally, authors' appreciation goes to all data collectors and supervisors who participated in the study.

\section{Competing interests}

The authors declare that they have no competing interests. 


\section{Availability of data and materials}

Data will be made available by the primary author upon request.

\section{Consent for publication}

Not applicable.

\section{Ethical approval and consent to participate}

Ethical clearance was obtained from the Institutional Review Board of the University of Gondar (Ref. No 550/09/07). The verbal consent procedures were approved by the Ethical review committee. An official permission letter was secured from the Dabat Research Center. All mothers were informed about the purpose of the study, and the interview was held only with those who gave verbal consent to participate. For mothers who were too young to make informed decision, consent was given on their behalf by a parent or legal guardian. The rights of participants to withdraw from the study at any time without any precondition were disclosed unequivocally. Moreover, records were locked and code numbers rather than personal identifiers were used to maintain confidentiality of information.

\section{Funding}

This study was funded by the University of Gondar. The views presented in the article are of the authors and do not necessarily express the views of the funding organization. The University of Gondar was not involved in the design of the study, data collection, analysis, and interpretation.

\section{Publisher's Note}

Springer Nature remains neutral with regard to jurisdictional claims in published maps and institutional affiliations.

Received: 23 December 2016 Accepted: 30 September 2017 Published online: 10 October 2017

\section{References}

1. World Health Organization: Global strategy on infant and young child feeding. World Health Organization; 2003.

2. Luan N-N, Wu Q-J, Gong T-T, Vogtmann E, Wang Y-L, Lin B. Breastfeeding and ovarian cancer risk: a meta-analysis of epidemiologic studies. Am J Clin Nutr. 2013;98:ajcn-062794.

3. Edmond KM, Kirkwood BR, Amenga-Etego S, Owusu-Agyei S, Hurt LS. Effect of early infant feeding practices on infection-specific neonatal mortality: an investigation of the causal links with observational data from rural Ghana. Am J Clin Nutr. 2007;86(4):1126-31.

4. World Health Organization: World alliance for breastfeeding action. World Health Organization; 2008.

5. Debes AK, Kohli A, Walker N, Edmond K, Mullany LC. Time to initiation of breastfeeding and neonatal mortality and morbidity: a systematic review. BMC public health. 2013;13(3):1.

6. Jones G, Steketee RW, Black RE, Bhutta ZA, Morris SS, Bellagio G. How many child deaths can we prevent this year? Lancet. 2003;362(9377):65-71.

7. Central Statistical Agency and ICF International. Ethiopia Demographic and Health Survey (EDHS) 2011. Addis Ababa, Ethiopia and Calverton: Central Statistical Agency [Ethiopia] and ICF International; 2012.

8. Lauer JA, Betran AP, Barros AJ, De Onis M. Deaths and years of life lost due to suboptimal breast-feeding among children in the developing world: a global ecological risk assessment. Public Health Nutr. 2006;9(6):673-85.

9. Bbaale E. Determinants of early initiation, exclusiveness, and duration of breastfeeding in Uganda. Int Cent Diarrhoeal Dis Res. 2014;32(2):249.

10. Okafor IP, Olatona FA, Olufemi OA. Breastfeeding practices of mothers of young children in Lagos, Nigeria. Niger J Paed. 2015;41(1):43-7.

11. Exavery A, Kanté AM, Hingora A, Phillips JF. Determinants of early initiation of breastfeeding in rural Tanzania. Int Breastfeed J. 2015;10:27.

12. Victor R, Baines SK, Agho KE, Dibley MJ. Determinants of breastfeeding indicators among children less than 24 months of age in Tanzania: a secondary analysis of the 2010 Tanzania Demographic and Health Survey. BMJ Open. 2013;3(1):e001529.
13. Joshi PC, Angdembe MR, Das SK, Ahmed S, Faruque ASG, Ahmed T. Prevalence of exclusive breastfeeding and associated factors among mothers in rural Bangladesh: a cross-sectional study. BMC Int Breastfeed J. 2014;9:7.

14. Adhikari M, Khanal V, Karkee R, Gavidia T. Factors associated with early initiation of breastfeeding among Nepalese mothers: further analysis of Nepal Demographic and Health Survey, 2011. Int Breastfeed J. 2014;9(1):21.

15. El-Gilany A-H, Sarraf B, Al-Wehady A. Factors associated with timely initiation of breastfeeding in Al-Hassa province, Saudi Arabia. East Mediterr Health J. 2012;18(3):250.

16. Vieira TO, Vieira GO, Giugliani ERJ, Mendes CM, Martins CC, Silva LR. Determinants of breastfeeding initiation within the first hour of life in a Brazilian population: Cross-sectional study. BMC Public Health. 2010;10:760.

17. Örün E, Songül SY, Madendaù Y, Üstünyurt-Eras Z, Kutluk U, Yurdakök K. Factors associated with breastfeeding initiation time in a Baby-Friendly Hospital. Turk J Pediatr. 2010;52(1):10.

18. Tamiru D, Belachew T, Loha E, Mohammed S. Sub-optimal breastfeeding of infants during the first six months and associated factors in rural communities of Jimma Arjo Woreda, Southwest Ethiopia. BMC Public Health. 2012;12:363.

19. Setegn T, Gerbaba M, Belachew T. Determinants of timely initiation of breastfeeding among mothers in Goba Woreda, South East Ethiopia: a cross sectional study. BMC Public Health. 2011;11:217.

20. Mekuria G, Edris M. Exclusive breastfeeding and associated factors among mothers in Debre Markos, Northwest Ethiopia: a cross-sectional study. BMC Int Breastfeed J. 2015;10(1):1.

21. Adugna DT. Women's perception and risk factors for delayed initiation of breastfeeding in Arba Minch Zuria, Southern Ethiopia. Int Breastfeed J. 2014;9:8.

22. Tsedeke W, Tadesse B, Eyasu E. Prevalence and determinants of timely initiation of breastfeeding among lactating mothers of urban dwellers in Western Ethiopia. Int J Food Sci Qual Manag. 2014;31:110.

23. Hailemariam TWAE. Sufa $A_{i}$ : predictors of early breastfeeding initiation amongmothers of children under 24 months of age in rural part of West Ethiopia. BMC Public Health. 1076;2015:15.

24. Acharya $P$, Khanal $V$. The effect of mother's educational status on early initiation of breastfeeding: further analysis of three consecutive Nepal Demographic and Health Surveys. BMC Public Health. 1069;2015:15.

25. FDREC: Summary and statistical report of the 2007 population and Housing census. In. Addis Ababa; 2008.

26. Bouanene I, ElMhamdi S, Sriha A, Bouslah A, Soltani M. Knowledge and practices of women in Monastir, Tunisia regarding breastfeeding. East Mediterr Health J. 2010;16(8):879-85.

27. Nkala TE, Msuya SE. Prevalence and predictors of exclusive breastfeeding among women in Kigoma region, Western Tanzania: a community based cross-sectional study. Int Breastfeed J. 2011;6:17.

28. Dorgham LS, Hafez SK, Kamhawy H, Hassan W. Assessment of initiation of breastfeeding, prevalence of exclusive breast feeding and their predictors in taif, KSA. Life Sci J. 2014;11:1.

29. Kimani-Murage EW, Madise NJ, Fotso J-C, Kyobutungi C, Mutua MK, Gitau $T M$, Yatich N. Patterns and determinants of breastfeeding and complementary feeding practices in urban informal settlements, Nairobi Kenya. BMC Public Health. 2011;11:396.

30. Fosu-Brefo R, Arthur E. Effect of timely initiation of breastfeeding on child health in Ghana. Health Econ Rev. 2015;5:8.

31. Esteves TMB, Daumas RP, Oliveira MI, Andrade CA, Leite IC. Factors associated to breastfeeding in the first hour of life: systematic review. Rev Saude Publica. 2014;48(4):697-708.

32. Health FroEMo: Health sector development program IV: 2010/112014/15. In: Tulane University Technical assistance program, Ethiopia and the US Department of health and human services/center for disease control and prevention (DHHS/CDC). 2010.

33. Ethiopia GotFDaRo: National Nutrition Program June 2013-June 2015.

34. Health FMo: Ethiopian National Strategy on Infant and Young Child Feeding. 2004.

35. Tariku A, Biks GA, Wassie MM, Gebeyehu A, Getie AA. Factors associated with prelacteal feeding in the rural population of northwest Ethiopia: a community cross-sectional study. Int Breastfeed J. 2016;11(1):1. 
36. Biks GA, Tariku A, Tessema GA. Effects of antenatal care and institutional delivery on exclusive breastfeeding practice in northwest Ethiopia: a nested case-control study. Int Breastfeed J. 2015;10(1):30.

37. Salasibew MM, Filteau S, Marchant T. Measurement of breastfeeding initiation: ethiopian mothers' perception about survey questions assessing early initiation of breastfeeding. Int Breastfeed J. 2014;9(1):1.

38. Raheem RA, Binns CW, Chih HJ, Sauer K. Determinants of the introduction of prelacteal feeds in the Maldives. Breastfeed Med. 2014;9(9):473-8.

39. Ahmed F, Rahman M, Alam M. Prelacteal feeding: influencing factors and relation to establishment of lactation. Bangladesh Med Res Counc Bull. 1996:22:60-4.

40. Meshram II, Laxmaiah A, Venkaiah K, Brahmam GNV. Impact of feeding and breastfeeding practices on the nutritional status of infants in a district of Andhra Pradesh, India. Natl Med J India. 2012;25(4):201-6.

41. Ali S, Ali SF, Imam AM, Ayub S, Billoo AG. Perception and practices of breastfeeding of infants 0-6 months in an urban and a semi-urban community in Pakistan: a cross-sectional study. J Pak Med Assoc. 2011;61(1):99.

42. Mannion CA, Hobbs AJ, McDonald SW, Tough SC. Maternal perceptions of partner support during breastfeeding. Int Breastfeed J. 2013;8(1):1.
43. Baş NG, Arıkan D. Effect of breastfeeding counseling before cesarean section on initiation time of breastfeeding and newborn's body temperature in Turkey. Nurs Prac Today. 2016;2(3):121-30.

44. Gami N, Mishra A, Kocher S. To study the effect of counseling on early initiation of breast feeding in the first hour of life. Glob J Med Res. 2014;13:2.

45. Kistin N, Abramson R, Dublin P. Effect of peer counselors on breastfeeding initiation, exclusivity, and duration among low-income urban women. J Human Lact. 1994;10(1):11-5.

46. Webber B, Elieson B, Gazak C, Stadler D. Effect of breastfeeding peer counseling on initiation and duration of breastfeeding among adolescent mothers. J Am Diet Assoc. 1998:98(9):A64.

47. Arora S, McJunkin C, Wehrer J, Kuhn P. Major factors influencing breastfeeding rates: mother's perception of father's attitude and milk supply. Pediatrics. 2000;106(5):e67.

48. Pisacane A, Continisio Gl, Aldinucci M, D'Amora S, Continisio P. A controlled trial of the father's role in breastfeeding promotion. Pediatrics. 2005; 116(4):e494-8.

49. Ulak M, Chandyo RK, Mellander L, Shrestha PS, Strand TA. Infant feedingpractices in Bhaktapur, Nepal: a cross-sectional, health facility based survey. Int Breastfeed J. 2012;7(1):1.

\section{Submit your next manuscript to BioMed Central and we will help you at every step:}

- We accept pre-submission inquiries

- Our selector tool helps you to find the most relevant journal

- We provide round the clock customer support

- Convenient online submission

- Thorough peer review

- Inclusion in PubMed and all major indexing services

- Maximum visibility for your research

Submit your manuscript at www.biomedcentral.com/submit
() Biomed Central 